\title{
Synthesis and Spectroscopic Characterization of Some New Biological Active Azo-Pyrazoline Derivatives
}

\author{
FAROUQ E. HAWAIZ AND MOHAMMAD K. SAMAD \\ Department of Chemistry, College of Education/Scientific Depts., \\ University of Salahaddin, Hawler, Kurdistan Region, Iraq \\ emamfarouq@yahoo.com
}

Received 09 November 2011; Accepted 15 January 2012

\begin{abstract}
A number of 3-[4-(benzyloxy)-3-(2-Chlorophenylazo)-phenyl]-5(substituted-phenyl)-1- substituted-2-pyrazolines( $4 a-j)$ and (5a-j) have been synthesized by diazotization of 2-chloroaniline and its coupling reaction with 4-hydroxy acetophenone, followed by benzyloxation of the hydroxyl group to give the substrate [4-benzyloxy-3-(2-chlorophenylazo)acetophenone (1)].The prepared starting material (1) has been reacted with different substituted benzaldehydes to give a new series of chalcone derivatives 1-[(4- benzyloxy)-3-(2-chloro-phenylazo) -phenyl]-3(substituted phenyl)-2-propen-1-one (3a-j), in high yields and in a few minutes, and the later compounds were treated with hydrazine hydrate according to Michael addition reaction to afford a new biolological active target compounds (4a-j) and (5a-j). Furthermore, The structures of the newly synthesized compounds were confirmed by FT-IR, ${ }^{13} \mathrm{C}-\mathrm{NMR},{ }^{13} \mathrm{C}-\mathrm{DEPT}$ $\&{ }^{1} \mathrm{H}-\mathrm{NMR}$ spectral data. The chalcone and pyrazoline derivatives were evaluated for their anti bacterial activity against Escherichia coli as gram negative and Staphylococcus aureus as gram positive, the results showed significant activity against both types of bacteria.
\end{abstract}

Keywords : diazotization, benzyloxation ,Chalcone, Pyrazoline, Anti-bacterial activity.

\section{Introduction}

Azo-coupling is one of the most important reactions for combining aromatic rings ${ }^{[1]}$ and preparing azobenzene derivatives containing active functional groups as a precursor for further synthesis to give different organic molecules such as : azo-amide ${ }^{[2]}$, azo-imine ${ }^{[3]}$ and azo- chalcone ${ }^{[4]}$. Azo compounds are important as synthetic dyes ${ }^{[5,6]}$ and cosmetics [7]. Chalcones and azo-chalcones are useful precursor for the synthesis of different heterocyclic compounds like pyrimidines ${ }^{[8]}$, thiazepines ${ }^{[9]}$ and Pyrazolines ${ }^{[10,12]}$. All derivatives are known to possess impressive biological activities. Such as: anti-malarial ${ }^{[13]}$, anti-bacterial ${ }^{[14]}$, anti-oxidative ${ }^{[15]}$, anti-fungal ${ }^{[16]}$, anti-leishmanial ${ }^{[17]}$, anti- tumour 
${ }^{[18]}$, central nervous system ${ }^{[19]}$, anti-histaminic ${ }^{[20]}$. Herein, we have described the synthesis of some new azo-pyrazoline compounds derived from 2-chloro aniline and phydroxy acetophenone with evaluation their anti bacterial studies.

\section{Experimental}

Melting points were determined using an Electrothermal melting point apparatus, IR spectra were recorded on a Bio-rad Merlin FT-IR spectroscopy Mod FTS 3000, using KBr disc. ${ }^{1} \mathrm{H}-\mathrm{NMR}$ and $\mathrm{C}^{13}$-NMR and ${ }^{13} \mathrm{C}$-DEPT-135 spectra were recorded on a Bruker(300MHz) with TMS as internal reference in( Jordon) :

\section{1-Preparation of 3-(2-chlorophenylazo)-4-hydroxyacetophenone (1) ${ }^{[21]}$}

The pure compound prepared according to the procedure ${ }^{[21]}$ as yellow crystals, of 3-(2chlorophenyl-azo)-4-hydroxyacetophenone (1). $\left(\mathrm{C}_{14} \mathrm{H}_{11} \mathrm{ClO}_{2} \mathrm{~N}_{2}\right)$, m.p. $\left(148-150 \mathrm{C}^{\circ}\right)$, yield of $(10.86 \mathrm{gm}, 99 \%) . \mathrm{IR}\left(\mathrm{cm}^{-1}\right)$ str. $3431(\mathrm{OH}), 1679(\mathrm{C}=\mathrm{O}), 1607(\mathrm{C}=\mathrm{C}), 1557(-\mathrm{N}=\mathrm{N}-)$, 1276 (C-O), ${ }^{1} \mathrm{H}-\mathrm{NMR}(\mathrm{ppm}): 2.68\left(\mathrm{~s}, 3 \mathrm{H}, \mathrm{H}_{1}-\mathrm{CH}_{3}\right) ; 7.12\left(\mathrm{~d}, 1 \mathrm{H}, \mathrm{H}_{5^{\prime}}\right) ; 7.41(\mathrm{~d}, 1 \mathrm{H}$, $\left.\mathrm{H}_{11}\right) ; 7.49\left(\mathrm{~d}, 1 \mathrm{H}, \mathrm{H}_{12}\right) ; 7.63\left(\mathrm{~d}, 1 \mathrm{H}, \mathrm{H}_{13}\right) ; 7.98\left(\mathrm{~d}, 1 \mathrm{H}, \mathrm{H}_{10}\right) ; 8.06\left(\mathrm{~d}, 1 \mathrm{H}, \mathrm{H}_{4}\right) ; 8.64$ $\left(\mathrm{s}, 1 \mathrm{H}, \mathrm{H}_{8}\right) 12.25(\mathrm{~s}, 1 \mathrm{H}, \mathrm{OH})$.

\section{2-Preparation of 3-(2-chlorophenylazo)-4-benzyloxyacetophen-one (2) ${ }^{[22]}$}

A mixture of 3-(2-chlorophenylazo)-4-hydroxy acetophenone (15gm, $0.0546 \mathrm{~mol})$, benzyl bromide (14.02gm, $0.082 \mathrm{~mol})$ and anhydrous $\mathrm{K}_{2} \mathrm{CO}_{3}$ (15.1 gm, $\left.0.109 \mathrm{~mol}\right)$ in ethanol (200 $\mathrm{ml}-96 \%$ ) was refluxed with stirring for $6 \mathrm{hrs}$. The cooled solution poured into water, solid materials immediately was obtained. The product was filtered off, washed several times with cold water, dried and recrystallized with a mixture $(1: 2)$ xylene: ethanol to obtain yellow-orange crystals of 3-(2-chlorophenylazo)- 4-benzyloxy acetophenone (2). $\left(\mathrm{C}_{21} \mathrm{H}_{17} \mathrm{ClO}_{2} \mathrm{~N}_{2}\right)$, m.p. (133-134C $\mathrm{C}^{\mathrm{o}}$ ), yield (11.9 gm, 59\%). IR $\left(\mathrm{cm}^{-1}\right)$ str, $1673(\mathrm{C}=\mathrm{O}), 1598$ $(\mathrm{C}=\mathrm{C}), 1532(-\mathrm{N}=\mathrm{N}-), 1271(\mathrm{C}-\mathrm{O}),{ }^{1} \mathrm{H}-\mathrm{NMR}(\mathrm{ppm}): 2.61\left(\mathrm{~s}, 3 \mathrm{H}, \mathrm{H}_{1}-\mathrm{CH}_{3}\right) ; 5.39(\mathrm{~s}, 2 \mathrm{H}$, $\left.\mathrm{H}_{15}\right) ; 7.16\left(\mathrm{~d}, 1 \mathrm{H}, \mathrm{H}_{5}\right) 7.35-7.51\left(\mathrm{~m}, 5 \mathrm{H}, \mathrm{H}_{17,18,19,20,21}\right) ; 7.62\left(\mathrm{~d}, 1 \mathrm{H}, \mathrm{H}_{11}\right) ; 7.71(\mathrm{~d}$, $\left.1 \mathrm{H}, \mathrm{H}_{12}\right) ; 7.82\left(\mathrm{~d}, 1 \mathrm{H}, \mathrm{H}_{13}\right) ; 7.91\left(\mathrm{~d}, 1 \mathrm{H}, \mathrm{H}_{10}\right) ; 8.04\left(\mathrm{~d}, 1 \mathrm{H}, \mathrm{H}_{4}\right) ; 8.45\left(\mathrm{~s}, 1 \mathrm{H}, \mathrm{H}_{8}\right)$. ${ }^{13}$ C-NMR : $\mathrm{C}_{1}: 26.38 ; \mathrm{C}_{15}: 71.28 ; \mathrm{C}_{5}: 116.91 ; \mathrm{C}_{8}: 119.06 ; \mathrm{C}_{10}: 126.13 ; \mathrm{C}_{11}: 127.90$; $\mathrm{C}_{17,21}: 128.48 ; \mathrm{C}_{14}: 129.22 ; \mathrm{C}_{19}: 129.59 ; \mathrm{C}_{18,20}: 130.48 ; \mathrm{C}_{13}: 131.08 ; \mathrm{C}_{12}: 131.52 ; \mathrm{C}_{3}$ : $132.83 ; \mathrm{C}_{4}: 133.49 ; \mathrm{C}_{7}: 135.39 ; \mathrm{C}_{16}: 136.13 ; \mathrm{C}_{9}: 142.14 ; \mathrm{C}_{6}: 159.84 ; \mathrm{C}_{2}: 196.7$. Dept-135: $\mathrm{C}_{1}: 26.38 ; \mathrm{C}_{15}: 71.28 ; \mathrm{C}_{5}: 116.91 ; \mathrm{C}_{8}: 119.06 ; \mathrm{C}_{10}: 126.13 ; \mathrm{C}_{11}: 127.90 ; \mathrm{C}_{17,21}: 128.48$ ; $\mathrm{C}_{19}: 129.59 ; \mathrm{C}_{18,20}: 130.48 ; \mathrm{C}_{13}: 131.08 ; \mathrm{C}_{12}: 131.52 ; \mathrm{C}_{4}: 133.49$.

\section{3- Synthesis of chalcones $(3 a-j)^{[23]}$ :-}

The prepared 3-(2-chlorophenylazo)-4-benzyloxyacetophenone (0.91 gm, $0.0025 \mathrm{~mol})$ was dissolved in $20 \mathrm{ml}$ of $96 \%$ ethanol, and added to the solution of an appropriate substituted benzaldehydes $(0.0025 \mathrm{~mol})$ in $96 \%$ ethanol $(20 \mathrm{ml})$ and $(2.5 \mathrm{ml})$ of $4 \%$ ethanolic sodium hydroxide. The mixture was stirred at room temperature for (1-5 min.) until the formation of pale yellow crystals of chalcone, and then kept the solution overnight at room temperature. The solid crystals were separated by suction filteration, washed with ethanol and water to neutralize, dried and purified by recrystallization from $(1: 2)$ xylene : ethanolthe results were illustrated in table (1) 
Table 1. Melting points and yields for the prepared chalcones (3a-j).

\begin{tabular}{|c|c|c|c|c|}
\hline Prod. & $\mathbf{R}$ & Molecular formula & M.P. $/{ }^{\mathbf{0}} \mathbf{C}$ & \% Yield \\
\hline $3 \mathrm{a}$ & $\mathrm{H}$ & $\mathrm{C}_{28} \mathrm{H}_{21} \mathrm{ClN}_{2} \mathrm{O}_{2}$ & $103-105$ & 85 \\
\hline $3 \mathrm{~b}$ & $2-\mathrm{F}$ & $\mathrm{C}_{28} \mathrm{H}_{20} \mathrm{ClFN}_{2} \mathrm{O}_{2}$ & $122-124$ & 86 \\
\hline $3 \mathrm{c}$ & $2-\mathrm{Cl}$ & $\mathrm{C}_{28} \mathrm{H}_{20} \mathrm{Cl}_{2} \mathrm{~N}_{2} \mathrm{O}_{2}$ & $128-130$ & 93 \\
\hline $3 \mathrm{~d}$ & $4-\mathrm{Cl}$ & $\mathrm{C}_{28} \mathrm{H}_{20} \mathrm{Cl}_{2} \mathrm{~N}_{2} \mathrm{O}_{2}$ & $138-140$ & 75 \\
\hline $3 \mathrm{e}$ & $4-\mathrm{F}$ & $\mathrm{C}_{28} \mathrm{H}_{20} \mathrm{ClF} \mathrm{N}_{2} \mathrm{O}_{2}$ & $161-163$ & 97 \\
\hline $3 \mathrm{f}$ & $4-\mathrm{Br}$ & $\mathrm{C}_{28} \mathrm{H}_{20} \mathrm{ClBrN}_{2} \mathrm{O}_{2}$ & $151-152$ & 86 \\
\hline $3 \mathrm{~g}$ & $4-\mathrm{CH}_{3}$ & $\mathrm{C}_{29} \mathrm{H}_{23} \mathrm{ClN}_{2} \mathrm{O}_{2}$ & $157-158$ & 60 \\
\hline $3 \mathrm{~h}$ & $4-\mathrm{OCH}_{3}$ & $\mathrm{C}_{29} \mathrm{H}_{23} \mathrm{Cl} \mathrm{N}_{2} \mathrm{O}_{3}$ & $104-106$ & 75 \\
\hline $3 \mathrm{i}$ & $3-\mathrm{O}-\mathrm{CH}_{2}-\mathrm{C}_{6} \mathrm{H}_{5}$ & $\mathrm{C}_{35} \mathrm{H}_{27} \mathrm{Cl}_{2} \mathrm{O}_{3}$ & $130-131$ & 76 \\
\hline $3 \mathrm{j}$ & $3-\left(4-\mathrm{Cl}_{2} \mathrm{C}_{6} \mathrm{H}_{4}-\right.$ & $\mathrm{C}_{35} \mathrm{H}_{26} \mathrm{Cl}_{2} \mathrm{~N}_{2} \mathrm{O}_{3}$ & $140-142$ & 84 \\
& $\left.\mathrm{CH}_{2} \mathrm{O}\right)$ & & & \\
\hline
\end{tabular}

3e: ${ }^{1} \mathrm{H}-\mathrm{NMR}$;5.48 (s 2H -O-CH $-\mathrm{C}_{22}$ ), 7.12 ( d 2H Ar-H-C ${ }_{3,5}$ ), 7.15 (d 1H Ar-H-C ${ }_{12}$ ), 7.197.56 ( $\mathrm{m}$ 7H Ar-H-C $2,6,24,25,26,27,28$ and $1 \mathrm{H} \mathrm{CH}-\mathrm{C} \alpha$ ), 7.69 ( d 2H Ar-H-C ${ }_{17,20}$ ), 7.91 (d 1H Ar$\mathrm{H}-\mathrm{C}_{11}$ ), 7.97 ( d $2 \mathrm{H} \mathrm{Ar}-\mathrm{H}-\mathrm{C}_{18,19}$ ). 8.18 (d $\left.1 \mathrm{H} \mathrm{CH}-\beta-\mathrm{C}_{7}\right), 8.4$ (s $\left.1 \mathrm{H} \mathrm{Ar}-\mathrm{H}-\mathrm{C}_{15}\right) .{ }^{13} \mathrm{C}-\mathrm{NMR}$, 71.38: $\mathrm{O}-\mathrm{CH}_{2} \mathrm{C}_{22}, \quad 114.81: \mathrm{C}_{12}, \quad 116.27: \mathrm{C}_{3,5}, \quad 118.03: \mathrm{CH}_{\alpha} \mathrm{C}_{8}, \quad 118.44: \mathrm{C}_{17}, 121.28: \mathrm{C}_{15}$, 127.07: $\mathrm{C}_{18}, 128.18: \mathrm{C}_{24,28}, 128.71: \mathrm{C}_{26}, 130.30: \mathrm{C}_{2,6}, 130.42: \mathrm{C}_{25,27}, 130.73: \mathrm{C}_{20}$, 131.21: $\mathrm{C}_{21}, 131.90: \mathrm{C}_{19}, 133.03: \mathrm{C}_{11}, 135.49: \mathrm{C}_{10}, 136.20: \mathrm{C}_{1}, 142.27: \mathrm{C}_{14}$ 143.28: $\mathrm{CH}_{\beta}$ $\mathrm{C}_{7}, 149.07: \mathrm{C}_{23} 159.69: \mathrm{C}_{16}, 162.39: \mathrm{C}_{4}, 165.72: \mathrm{C}_{13}, \quad 188.36: \mathrm{C}=\mathrm{O} \mathrm{C}_{9} ;{ }^{13} \mathrm{C}-\mathrm{DEPT}-$ 71.38: $\mathrm{O}_{-} \mathrm{CH}_{2} \mathrm{C}_{22}, \quad 114.81: \mathrm{C}_{12}, \quad 116.27: \mathrm{C}_{3,5}, \quad 118.03: \mathrm{CH}_{\alpha} \mathrm{C}_{8}, \quad 118.44: \mathrm{C}_{17}, 121.28: \mathrm{C}_{15}$, 127.07: $\mathrm{C}_{18}, 128.18: \mathrm{C}_{24,28}, 128.71: \mathrm{C}_{26}, 130.30: \mathrm{C}_{2,6}, 130.42: \mathrm{C}_{25,27}, 130.73: \mathrm{C}_{20}$, 131.90: $\mathrm{C}_{19}, 133.03: \mathrm{C}_{11}, 143.28: \mathrm{CH}_{\beta} \mathrm{C}_{7}$.

Table 2. Reaction times, melting points and yields for the prepared pyrazolines (4a-j).

\begin{tabular}{|c|c|c|c|c|c|}
\hline Prod. & $\mathbf{R}$ & $\begin{array}{c}\text { Molecular } \\
\text { formula }\end{array}$ & Time/hrs. & M.P. ${ }^{\mathbf{0} C}$ & \% Yield \\
\hline $4 \mathrm{a}$ & $\mathrm{H}$ & $\mathrm{C}_{28} \mathrm{H}_{23} \mathrm{ClN}_{4} \mathrm{O}$ & 1.25 & $95-97$ & 78 \\
\hline $4 \mathrm{~b}$ & $2-\mathrm{F}$ & $\mathrm{C}_{28} \mathrm{H}_{22} \mathrm{ClFN}_{4} \mathrm{O}$ & 0.75 & $113-114$ & 83 \\
\hline $4 \mathrm{c}$ & $2-\mathrm{Cl}$ & $\mathrm{C}_{28} \mathrm{H}_{22} \mathrm{ClFN}_{4} \mathrm{O}$ & 0.5 & $143-144$ & 98 \\
\hline $4 \mathrm{~d}$ & $4-\mathrm{Cl}$ & $\mathrm{C}_{28} \mathrm{H}_{22} \mathrm{Cl}_{2} \mathrm{~N}_{4} \mathrm{O}$ & 0.75 & $116-118$ & 84 \\
\hline $4 \mathrm{e}$ & $4-\mathrm{F}$ & $\mathrm{C}_{28} \mathrm{H}_{22} \mathrm{ClF} \mathrm{N}_{4} \mathrm{O}$ & 0.75 & $137-139$ & 77 \\
\hline $4 \mathrm{f}$ & $4-\mathrm{Br}$ & $\mathrm{C}_{28} \mathrm{H}_{22} \mathrm{ClBrN}_{4} \mathrm{O}$ & 1 & $90-92$ & 71 \\
\hline $4 \mathrm{~g}$ & $4-\mathrm{CH}_{3}$ & $\mathrm{C}_{29} \mathrm{H}_{25} \mathrm{ClN}_{4} \mathrm{O}$ & 0.5 & $107-109$ & 61 \\
\hline $4 \mathrm{~h}$ & $4-\mathrm{OCH}_{3}$ & $\mathrm{C}_{29} \mathrm{H}_{25} \mathrm{Cl} \mathrm{N}_{4} \mathrm{O}_{2}$ & 1 & $66-68$ & 79 \\
\hline $4 \mathrm{i}$ & $3-\mathrm{O}-\mathrm{CH}_{2}-\mathrm{C}_{6} \mathrm{H}_{5}$ & $\mathrm{C}_{35} \mathrm{H}_{29} \mathrm{Cl} \mathrm{N}_{4} \mathrm{O}_{2}$ & 1 & $115-116$ & 81 \\
\hline $4 \mathrm{j}$ & $\begin{array}{c}3-\left(4-\mathrm{Cl}_{2} \mathrm{C}_{6} \mathrm{H}_{4}-\right. \\
\left.\mathrm{CH}_{2} \mathrm{O}\right)\end{array}$ & $\mathrm{C}_{35} \mathrm{H}_{28} \mathrm{Cl}_{2} \mathrm{~N}_{4} \mathrm{O}_{2}$ & 1 & $95-97$ & 86 \\
\hline
\end{tabular}

3g: ${ }^{1} \mathrm{H}-\mathrm{NMR} ; 2.41$ (s $3 \mathrm{H}-\mathrm{CH}_{3}-\mathrm{C}_{29}$ ), 5.45 (s $2 \mathrm{H}-\mathrm{O}-\mathrm{CH}_{2}-\mathrm{C}_{22}$ ) 7.12-7.9 (m 15H Ar-H$\mathrm{C}_{2,3,5,6,11,12,17,18,19,20,24,25,26,27,28}$ and $1 \mathrm{H} \mathrm{CH}-\mathrm{C} \alpha$ ), 8.19 (d $\left.1 \mathrm{H} \mathrm{CH}-\beta-\mathrm{C}_{7}\right), 8.41$ (s $1 \mathrm{H} \mathrm{Ar}-\mathrm{H}-$ $\mathrm{C}_{15}$ ); 21.57:- $\mathrm{CH}_{3},{ }^{13} \mathrm{C}-\mathrm{NMR} 71.33: \mathrm{O}-\mathrm{CH}_{2} \mathrm{C}_{22}, 114.73: \mathrm{C}_{12}, 118.04: \mathrm{CH}_{\alpha} \mathrm{C}_{8}, 118.43: \mathrm{C}_{17}$, $120.54: \mathrm{C}_{15}$ 126.04: $\mathrm{C}_{2,6}, 127.07: \mathrm{C}_{18}, 127.36: \mathrm{C}_{24,28}, 128.16: \mathrm{C}_{26}, 128,87: \mathrm{C}_{21}, 128.92:$ $\mathrm{C}_{25,27}, 129.73: \mathrm{C}_{3,5}, 130.73: \mathrm{C}_{20}, 131.45: \mathrm{C}_{10}, 131.87: \mathrm{C}_{19}, 132.20: \mathrm{C}_{1}, 133.06: \mathrm{C}_{11}$, , 
136.2: $\mathrm{C}_{4}, 141.08: \mathrm{C}_{14}, 142.25: \mathrm{C}_{23}, \quad$ 144.75: $\mathrm{CH}_{\beta} \mathrm{C}_{7}, \quad$ 149.08: $\mathrm{C}_{16}, \quad$ 159.58: $\mathrm{C}_{13}$, $188.54: \mathrm{C}=\mathrm{O} \mathrm{C}{ }_{9 ;}{ }^{13} \mathrm{C}-\mathrm{DEPT}$

3j: ${ }^{1} \mathrm{H}-\mathrm{NMR} ; 5.09$ (s $2 \mathrm{H}-\mathrm{O}-\mathrm{CH}_{2}-\mathrm{C}_{22}$ ), 5.45 (s $2 \mathrm{H}-\mathrm{O}-\mathrm{CH}_{2}-\mathrm{C}_{29}$ ), 7.04 (d 1H Ar-H-C $\mathrm{C}_{12}$ ),

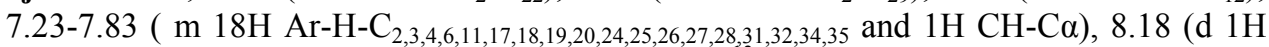
$\mathrm{CH}-\beta-\mathrm{C}_{7}$ ), 8.45 (s 1H Ar-H-C 15 ); 69.32 :O- $\mathrm{CH}_{2} \mathrm{C}_{22},{ }^{13} \mathrm{C}-\mathrm{NMR}, 71.38: \mathrm{O}-\mathrm{CH}_{2} \mathrm{C}_{29},, 114.07$ $: \mathrm{C}_{6}, 114.82: \mathrm{C}_{4}, 117.29: \mathrm{C}_{12}, 118.02: \mathrm{C}_{2} 118.51: \mathrm{CH}_{\alpha} \mathrm{C}_{8}, 121.75: \mathrm{C}_{17}, 121.94: \mathrm{C}_{15}$, $127.08: \mathrm{C}_{18}, 127.39: \mathrm{C}_{24,26,28}, 128.18: \mathrm{C}_{25,27} 128.72: \mathrm{C}_{31,35}, 128.82: \mathrm{C}_{32,34}, 130.06: \mathrm{C}_{20}$, 130.73: $\mathrm{C}_{3}, 131.22: \mathrm{C}_{10}, 131.92: \mathrm{C}_{19}, 1133.06: \mathrm{C}_{11}, 133.89: \mathrm{C}_{21}, 135.21: \mathrm{C}_{33}, 135.51: \mathrm{C}_{1}$, 136.17: $C_{14}, 136.43: C_{23}, 142.26: C_{30}, 144.29: \mathrm{CH}_{\beta} C_{7}, 149.02: C_{16}, 158.91: C_{5}, 159.71$ : $\mathrm{C}_{13}, 188.43: \mathrm{C}=\mathrm{O} \mathrm{C}_{9} ;{ }^{13} \mathrm{C}-\mathrm{DEPT}$

4- Synthesis of pyrazolines 3-[4-(benzyloxy)-3-(2-chlorophenylazo)-phenyl]-5(substituted-phenyl)-1-substituted-2-pyrazolines( $4 a-j)$ and $(5 a-j)^{[24]}$ :

A mixture of chalcone derivative $(0.5 \mathrm{mmoles})$, hydrazine hydrate $(2.5 \mathrm{mmoles})$ or phenylhydrazine (1mmoles) and sodium hydroxide $(5 \mathrm{ml}, 0.4 \%)$ in ethanol $(15 \mathrm{ml})$ was refluxed with stirring for appropriate time until complete the reaction, which was monitored by either change of the color or the formation of ppt. The ppt. was isolated by suction filteration, washed with ethanol and water to neutralize, dried and purified by recrystallization from (1:2) xylene: ethanol. The physical properties and yields are summarized in table- 2 .

4e: ${ }^{1} \mathrm{H}-\mathrm{NMR} ; 3.06$ (dd $1 \mathrm{H} \mathrm{CH} \mathrm{CH}_{2}-\mathrm{H}_{\mathrm{a}}-\mathrm{C}_{8}$ ), 3.52 (dd $\left.1 \mathrm{H} \mathrm{CH} \mathrm{CH}_{2}-\mathrm{H}_{\mathrm{b}}-\mathrm{C}_{8}\right), 4.93$ (dd $1 \mathrm{H} \mathrm{CH}-\mathrm{H}_{\mathrm{x}}-$ $\left.\mathrm{C}_{7}\right), 5.43\left(\mathrm{~S} 2 \mathrm{H}-\mathrm{O}-\mathrm{CH}_{2}-\mathrm{C}_{22}\right), 5.95$ br.s $\left.1 \mathrm{H} \mathrm{N}-\mathrm{H}\right), \quad 7.01-7.93$ (m $\left.16 \mathrm{H} \mathrm{Ar}-\mathrm{H}\right) ;{ }^{13} \mathrm{C}-\mathrm{NMR}$ ;41.5: $\mathrm{CH}_{2} \mathrm{C}_{8}$, 63.79: $\mathrm{CH} \mathrm{C}_{7}$, 71.66: $\mathrm{O}-\mathrm{CH}_{2} \mathrm{C}_{22}$, 115.38: $\mathrm{C}_{12}, 115.5: \mathrm{C}_{3,5}, 115.79: \mathrm{C}_{17}$, 118.05: $\mathrm{C}_{15}, 126.27: \mathrm{C}_{10}$, 127.10: $\mathrm{C}_{18}$, 127.30: $\mathrm{C}_{24,28}, 127.99: \mathrm{C}_{26}, 128.10: \mathrm{C}_{2,6}$, 128.61: $\mathrm{C}_{25,27}, 130.24: \mathrm{C}_{20}, 130.61: \mathrm{C}_{19}, 131.56: \mathrm{C}_{11}, 138.69: \mathrm{C}_{21}, 138.42: \mathrm{C}_{1}, 142.61: \mathrm{C}_{14}$, 149.18: $\mathrm{C}_{23}, 150.56: \mathrm{C}_{16}, 157.05: \mathrm{C}_{9}, \quad 160.69: \mathrm{C}_{13}, 163.95: \mathrm{C}_{4} ;{ }^{13} \mathrm{C}-\mathrm{DEPT} ;-41.5: \mathrm{CH}_{2}$ $\mathrm{C}_{8}$, 63.79: $\mathrm{CH} \mathrm{C}$, 71.66: $\mathrm{O}-\mathrm{CH}_{2} \mathrm{C}_{22}, 115.38: \mathrm{C}_{12}, 115.5: \mathrm{C}_{3,5}, 115.79: \mathrm{C}_{17}, 118.05: \mathrm{C}_{15}$, 127.10: $\mathrm{C}_{18}, 127.30: \mathrm{C}_{24,28}, 127.99: \mathrm{C}_{26}, 128.10: \mathrm{C}_{2,6}, 128.61: \mathrm{C}_{25,27}, 130.24: \mathrm{C}_{20}$, 130.61: $\mathrm{C}_{19}, 131.56: \mathrm{C}_{11}$.

4g: ${ }^{1} \mathrm{H}-\mathrm{NMR} ; 2.30$ (s $3 \mathrm{H} \mathrm{CH}-\mathrm{C}_{29}$ ), 3.09 (dd $1 \mathrm{H} \mathrm{CH}_{2}-\mathrm{H}_{\mathrm{a}}-\mathrm{C}_{8}$ ), 3.58 (dd $1 \mathrm{H} \mathrm{CH}_{2}-\mathrm{H}_{\mathrm{b}}-\mathrm{C}_{8}$ ), 5.25 (dd $\left.1 \mathrm{H} \mathrm{CH}-\mathrm{H}_{\mathrm{x}}-\mathrm{C}_{7}\right), 5.39$ (s $\left.2 \mathrm{H}-\mathrm{O}-\mathrm{CH}_{2}-\mathrm{C}_{22}\right), 5.92$ (br.s $\left.1 \mathrm{H} \mathrm{N}-\mathrm{H}\right), 6.89-7.95(\mathrm{~m} 16 \mathrm{H}$ $\mathrm{Ar}-\mathrm{H}) ;{ }^{13} \mathrm{C}-\mathrm{NMR} ; 21.52: \mathrm{CH}_{3} \mathrm{C}_{29}, 41.45: \mathrm{CH} \mathrm{C}$, 63.3: $\mathrm{CH} \quad \mathrm{C}_{7}, 71.43: \mathrm{O}_{-} \mathrm{CH}_{2} \mathrm{C}_{22}$, 114.89: $\mathrm{C}_{12}, 118.58: \mathrm{C}_{17}, 119.54: \mathrm{C}_{15}, 125.76: \mathrm{C}_{10}, 126.16: \mathrm{C}_{18}, 126.93: \mathrm{C}_{2,6}$, 127.11: $\mathrm{C}_{24,28}, \quad 127.23: \mathrm{C}_{26}, 128.56: \mathrm{C}_{25,27}, \quad 129.09: \mathrm{C}_{3,5}, 129.95: \mathrm{C}_{20}, \quad 130.45: \mathrm{C}_{19}$, 131.65: $\mathrm{C}_{11}, 132.92: \mathrm{C}_{21}, 136.87: \mathrm{C}_{4}, 138.18: \mathrm{C}_{14}, 140.23: \mathrm{C}_{1}, 144.24: \mathrm{C}_{23}, 149.69: \mathrm{C}_{16}$, 152.02: $\mathrm{C}_{9}, 161.38: \mathrm{C}_{13} \cdot{ }^{13} \mathrm{C}-\mathrm{DEPT} ;-21.52: \mathrm{CH}_{3} \mathrm{C}_{29}, 41.45: \mathrm{CH} \mathrm{C}$, 63.3: $\mathrm{CH} \mathrm{C}_{7}, 71.43:$ $\mathrm{O}_{-} \mathrm{CH}_{2} \quad \mathrm{C}_{22}, 114.89: \mathrm{C}_{12}, 118.58: \mathrm{C}_{17}, 119.54: \mathrm{C}_{15}, 126.16: \mathrm{C}_{18}, 126.93: \mathrm{C}_{2,6}$, 127.11: $\mathrm{C}_{24,28}, \quad 127.23: \mathrm{C}_{26}, 128.56: \mathrm{C}_{25,27}, 129.09: \mathrm{C}_{3,5}, 129.95: \mathrm{C}_{20}, 130.45: \mathrm{C}_{19}$, 131.65: $\mathrm{C}_{11}$.

4j: ${ }^{1} \mathrm{H}-\mathrm{NMR} ; 3.05$ (dd $1 \mathrm{H} \mathrm{CH}_{2}-\mathrm{H}_{\mathrm{a}}-\mathrm{C}_{8}$ ), 3.55 dd $1 \mathrm{H} \mathrm{CH}_{2}-\mathrm{H}_{\mathrm{b}}-\mathrm{C}_{8}$ ), 4.95 dd $1 \mathrm{H} \mathrm{CH}-\mathrm{H}_{\mathrm{x}}-\mathrm{C}_{7}$ ), 5.03 (d $\left.2 \mathrm{H}, 1 \mathrm{H}-\mathrm{O}-\mathrm{CH}_{2}-\mathrm{C}_{29}\right), 5.40\left(\mathrm{~d} 2 \mathrm{H}, 1 \mathrm{H}-\mathrm{O}-\mathrm{CH}_{2}-\mathrm{C}_{22}\right), 6.00$ (br.s $\left.1 \mathrm{H} \mathrm{N}-\mathrm{H}\right), 6.88-$ 8.04 (m 20H Ar-H41.5: $\mathrm{CH}_{2} \mathrm{C}_{8},{ }^{13} \mathrm{C}-\mathrm{NMR} ;$ 64.27: $\mathrm{CH} \mathrm{C}_{7}, 69.19: \mathrm{O}-\mathrm{CH}_{2} \mathrm{C}_{22}, 71.65: \mathrm{O}-$ $\mathrm{CH}_{2} \quad \mathrm{C}_{29}, 112.64: \mathrm{C}_{4}, 114.15: \mathrm{C}_{6}, 115.38: \mathrm{C}_{12}, 115.67: \mathrm{C}_{2}, 118.06: \mathrm{C}_{17}, 119.19: \mathrm{C}_{15}$, 126.35: $\mathrm{C}_{10}, 127.12: \mathrm{C}_{18}, \quad 127.31: \mathrm{C}_{24,28}, 128: \mathrm{C}_{26}, 128.62: \mathrm{C}_{25,27}, 128.75: \mathrm{C}_{31,35}$ ,128.79: $\mathrm{C}_{32,34}, 130.24: \mathrm{C}_{20}, 129.97: \mathrm{C}_{3}, 130.63: \mathrm{C}_{19}, 131.57: \mathrm{C}_{11}, 133.75: \mathrm{C}_{21}, 135.26: \mathrm{C}_{33}$, 135.39: $\mathrm{C}_{14} \quad 136.72: \mathrm{C}_{30} \quad 142.61: \mathrm{C}_{23}, 144.576: \mathrm{C}_{1}, 149.19: \mathrm{C}_{16}, 150.52: \mathrm{C}_{9}, \quad 153.03: \mathrm{C}_{13}$, 158.97: $\mathrm{C}_{5} ;{ }^{13} \mathrm{C}$ - DEPT; - 41.5: $\mathrm{CH}_{2} \mathrm{C}_{8},-64.27: \mathrm{CH} \mathrm{C}_{7},-69.19: \mathrm{O}_{-} \mathrm{CH}_{2} \mathrm{C}_{22},-71.65: \mathrm{O}-\mathrm{CH}_{2}$ $\mathrm{C}_{29}, 112.64: \mathrm{C}_{4}, 114.15: \mathrm{C}_{6}, 115.38: \mathrm{C}_{12}, 115.67: \mathrm{C}_{2}, 118.06: \mathrm{C}_{17}, 119.19: \mathrm{C}_{15}, 127.12: \mathrm{C}_{18}$, 127.31: $\mathrm{C}_{24,28}, 128: \mathrm{C}_{26}, 128.62: \mathrm{C}_{25,27}, 128.75: \mathrm{C}_{31,35}, 128.79: \mathrm{C}_{32,34}, 130.24: \mathrm{C}_{20}, 129.97: \mathrm{C}_{3}$, $130.63: \mathrm{C}_{19}, 131.57: \mathrm{C}_{11}$. 
Table 3. Reaction times, melting points and yields for the prepared pyrazolines $(5 \mathrm{a}-\mathrm{j})$.

\begin{tabular}{|c|c|c|c|c|c|}
\hline Prod. & $\mathbf{R}$ & $\begin{array}{c}\text { Molecular } \\
\text { formula }\end{array}$ & Time/hrs. & M.P. $\left.\right|^{0} \mathrm{C}$ & \%Yield \\
\hline $5 \mathrm{a}$ & $\mathrm{H}$ & $\mathrm{C}_{34} \mathrm{H}_{28} \mathrm{ClN}_{4} \mathrm{O}$ & 1.5 & $155-156$ & 46 \\
\hline $5 \mathrm{~b}$ & $2-\mathrm{F}$ & $\mathrm{C}_{34} \mathrm{H}_{22} \mathrm{ClFN}_{4} \mathrm{O}$ & 2 & $184-186$ & 45 \\
\hline $5 \mathrm{c}$ & $2-\mathrm{Cl}$ & $\mathrm{C}_{34} \mathrm{H}_{22} \mathrm{ClFN}_{4} \mathrm{O}$ & 1.25 & $183-184$ & 60 \\
\hline $5 \mathrm{~d}$ & $4-\mathrm{Cl}$ & $\mathrm{C}_{34} \mathrm{H}_{22} \mathrm{Cl}_{2} \mathrm{~N}_{4} \mathrm{O}$ & 2 & $180-182$ & 46 \\
\hline $5 \mathrm{e}$ & $4-\mathrm{F}$ & $\mathrm{C}_{34} \mathrm{H}_{22} \mathrm{ClF} \mathrm{N}_{4} \mathrm{O}$ & 1.5 & 189-191 & 48 \\
\hline $5 \mathrm{f}$ & $4-\mathrm{Br}$ & $\mathrm{C}_{34} \mathrm{H}_{22} \mathrm{ClBrN}_{4} \mathrm{O}$ & 1.25 & $140-141$ & 50 \\
\hline $5 \mathrm{~g}$ & $4-\mathrm{CH}_{3}$ & $\mathrm{C}_{35} \mathrm{H}_{25} \mathrm{ClN}_{4} \mathrm{O}$ & 3 & $189-190$ & 62 \\
\hline $5 \mathrm{~h}$ & $4-\mathrm{OCH}_{3}$ & $\mathrm{C}_{35} \mathrm{H}_{25} \mathrm{Cl} \mathrm{N} \mathrm{O}_{2}$ & 1 & $95-96$ & 44 \\
\hline $5 \mathrm{i}$ & $3-\mathrm{O}-\mathrm{CH}_{2}-\mathrm{C}_{6} \mathrm{H}_{5}$ & $\mathrm{C}_{41} \mathrm{H}_{33} \mathrm{Cl} \mathrm{N}_{4} \mathrm{O}_{2}$ & 2 & $125-126$ & 68 \\
\hline $5 \mathrm{j}$ & $\begin{array}{c}3-\left(4-\mathrm{Cl}_{-}-\mathrm{C}_{6} \mathrm{H}_{4}-\right. \\
\left.\mathrm{CH}_{2} \mathrm{O}\right)\end{array}$ & $\mathrm{C}_{41} \mathrm{H}_{32} \mathrm{Cl}_{2} \mathrm{~N}_{4} \mathrm{O}_{2}$ & 2 & $148-149$ & 52 \\
\hline
\end{tabular}

5e: ${ }^{1} \mathrm{H}-\mathrm{NMR} ; 3.09$ (dd $1 \mathrm{H} \mathrm{CH} \mathrm{CH}_{2}-\mathrm{H}_{\mathrm{a}}-\mathrm{C}_{8}$ ), 3.82 (dd $1 \mathrm{H} \mathrm{CH} \mathrm{CH}_{2}-\mathrm{H}_{\mathrm{b}}-\mathrm{C}_{8}$ ), 5.22 (dd $1 \mathrm{H} \mathrm{CH}-\mathrm{H}_{\mathrm{x}}-$ $\mathrm{C}_{7}$ ), 5.36 (S $2 \mathrm{H}-\mathrm{O}-\mathrm{CH}_{2}-\mathrm{C}_{22}$ ), 7.01-7.97 (m 21H Ar-H); ${ }^{13} \mathrm{C}-\mathrm{NMR} 43.04: \mathrm{CH}_{2} \mathrm{C}_{8}, 63.89$ : $\mathrm{CH} \mathrm{C}$, 71.65: $\mathrm{O}-\mathrm{CH}_{2} \mathrm{C}_{22}, 113.32: \mathrm{C}_{30,34}, 114.91: \mathrm{C}_{12}, 115.76: \mathrm{C}_{3,5}, 118.04: \mathrm{C}_{17}, 119.18$ : $\mathrm{C}_{15}, 126.13: \mathrm{C}_{10}, 127.10: \mathrm{C}_{18}, 127.49: \mathrm{C}_{24,28}, 127.60: \mathrm{C}_{26}, 127.98: \mathrm{C}_{25,27}, 128.58: \mathrm{C}_{2,6}$, $128.90: \mathrm{C}_{20}, 130.13: \mathrm{C}_{31,33}, 130.57: \mathrm{C}_{19}, 131.59: \mathrm{C}_{11}, 136.59: \mathrm{C}_{21}, 138.19: \mathrm{C}_{1}, 142.58: \mathrm{C}_{14}$, 144.67: $\mathrm{C}_{23}, 146.09: \mathrm{C}_{29}, 149.13: \mathrm{C}_{16}, 156.88: \mathrm{C}_{9}, \quad 160.46: \mathrm{C}_{13}, 163.71: \mathrm{C}_{4} ;{ }^{13} \mathrm{C}-\mathrm{DEPT}$; -43.04: $\mathrm{CH}_{2} \mathrm{C}_{8}$, 63.89: $\mathrm{CH} \mathrm{C}_{7},-71.65: \mathrm{O}_{-} \mathrm{CH}_{2} \mathrm{C}_{22}$, 113.32: $\mathrm{C}_{30,34}, 114.91: \mathrm{C}_{12}, 115.76$ $: \mathrm{C}_{3,5}, 118.04: \mathrm{C}_{17}, 119.18: \mathrm{C}_{15}, 127.10: \mathrm{C}_{18}, 127.49: \mathrm{C}_{24,28}, 127.60: \mathrm{C}_{26}, 127.98: \mathrm{C}_{25,27}$, 128.58: $\mathrm{C}_{2,6}, 128.90: \mathrm{C}_{20}, 130.13: \mathrm{C}_{31,33}, 130.57: \mathrm{C}_{19}, 131.59: \mathrm{C}_{11}$.

5g: ${ }^{1} \mathrm{H}-\mathrm{NMR}$;2.35 (s $3 \mathrm{H} \mathrm{CH}_{3}-\mathrm{C}_{29}$ ), 3.15 (dd $1 \mathrm{H} \mathrm{CH}_{2}-\mathrm{H}_{\mathrm{a}}-\mathrm{C}_{8}$ ), 3.85 (dd $1 \mathrm{H} \mathrm{CH}_{2}-\mathrm{H}_{\mathrm{b}}-\mathrm{C}_{8}$ ), 5.25 (dd $1 \mathrm{H} \mathrm{CH}-\mathrm{H}_{\mathrm{x}}-\mathrm{C}_{7}$ ), 5.41 (s $2 \mathrm{H}-\mathrm{O}-\mathrm{CH}_{2}-\mathrm{C}_{22}$ ), 6.80-7.99 ( m 21H Ar-H); ${ }^{13} \mathrm{C}-\mathrm{NMR}$ ;21.11: $\mathrm{CH}_{3} \mathrm{C}_{29}, 43.68: \mathrm{CH} \mathrm{C}_{8}, \quad 64.43: \mathrm{CH} \mathrm{C}_{7}, 71.73: \mathrm{O}^{-} \mathrm{CH}_{2} \mathrm{C}_{22}, 113.35: \mathrm{C}_{31,35}, 114.94$ : $\mathrm{C}_{12}, \quad 115.83: \mathrm{C}_{33}, 118.08: \mathrm{C}_{17}, 118.99: \mathrm{C}_{15}, 125.84: \mathrm{C}_{10}, 126.04: \mathrm{C}_{18}, 127.13: \mathrm{C}_{2,6}$, 127.31: $\mathrm{C}_{24,28}, \quad 127.99: \mathrm{C}_{26}, 128.62: \mathrm{C}_{25,27}, \quad 128.89: \mathrm{C}_{3,5}, 129.82: \mathrm{C}_{20}, 130.14:$ $\mathrm{C}_{32,34}, 130.63: \mathrm{C}_{19}, \quad 131.54: \mathrm{C}_{11}, 135.12: \mathrm{C}_{21} \quad, 136.56: \mathrm{C}_{4}, 137.11: \mathrm{C}_{14}, 139.45: \mathrm{C}_{1}$, 142.54: $\mathrm{C}_{23}, 144.76: \mathrm{C}_{30}, 146.09: \mathrm{C}_{16}, 149.12: \mathrm{C}_{9}, 156.68: \mathrm{C}_{13} \cdot{ }^{13} \mathrm{C}-\mathrm{DEPT} ; 21.11: \mathrm{CH}_{3} \mathrm{C}_{29}$, -43.68: $\mathrm{CH}_{2} \mathrm{C}_{8}$, 64.43: $\mathrm{CH} \mathrm{C}_{7},-71.73: \mathrm{O}_{-} \mathrm{CH}_{2} \mathrm{C}_{22}, 113.35: \mathrm{C}_{31,35}, 114.94: \mathrm{C}_{12}, 115.83:$ $\mathrm{C}_{33}, 118.08: \mathrm{C}_{17}, 118.99: \mathrm{C}_{15}, 126.04: \mathrm{C}_{18}, 127.13: \mathrm{C}_{2,6}, 127.31: \mathrm{C}_{24,28}, 127.99: \mathrm{C}_{26}$, 128.62: $\mathrm{C}_{25,27}, 128.89: \mathrm{C}_{3,5}, 129.82: \mathrm{C}_{20}, 130.14: \mathrm{C}_{32,34}, 130.63: \mathrm{C}_{19}, 131.54: \mathrm{C}_{11}$.

5j: ${ }^{1} \mathrm{H}-\mathrm{NMR}$; 3.15 (dd $1 \mathrm{H} \mathrm{CH} \mathrm{CH}_{2}-\mathrm{H}_{\mathrm{a}}-\mathrm{C}_{8}$ ), 3.87 dd $1 \mathrm{H} \mathrm{CH} \mathrm{CH}_{2}-\mathrm{H}_{\mathrm{b}}-\mathrm{C}_{8}$ ), 4.96 (d 2H, 1H -O$\left.\mathrm{CH}_{2}-\mathrm{C}_{29}\right), 5.41\left(\mathrm{dd} 1 \mathrm{H} \mathrm{CH}-\mathrm{H}_{\mathrm{x}}-\mathrm{C}_{7}\right.$ ), 5.90 (d 2H, $\left.1 \mathrm{H}-\mathrm{O}-\mathrm{CH}_{2}-\mathrm{C}_{22}\right), 6.84-8.09$ (m 25H Ar$\mathrm{H}) ;{ }^{13} \mathrm{C}-\mathrm{NMR} ; 43.56: \mathrm{CH}_{2} \mathrm{C}_{8}$, 64.56: $\mathrm{CH} \quad \mathrm{C}_{7}, 69.16: \mathrm{O}-\mathrm{CH}_{2} \mathrm{C}_{22}, 71.69: \mathrm{O}-\mathrm{CH}_{2} \mathrm{C}_{29}$, 112.11: $\mathrm{C}_{4}, 113.31: \mathrm{C}_{37,41}, 114.05: \mathrm{C}_{6}, 114.94: \mathrm{C}_{12}, 118.09: \mathrm{C}_{2}, 118.67: \mathrm{C}_{17}, 119.13: \mathrm{C}_{15}$, 126.25: $\mathrm{C}_{10}, \quad 127.12: \mathrm{C}_{18}$, 127.34: $\mathrm{C}_{24,28}, 128: 01 \mathrm{C}_{26}, 128.63: \mathrm{C}_{25,27}, 128.70: \mathrm{C}_{31,35}$, 128.89: $\mathrm{C}_{32,34}, 130.93: \mathrm{C}_{20}, 130.15: \mathrm{C}_{3}, 130.63: \mathrm{C}_{19}, 131.58: \mathrm{C}_{11}, 133.72: \mathrm{C}_{21}, 135.21: \mathrm{C}_{33}$, 135.26: $\mathrm{C}_{14} \quad, 136.70: \mathrm{C}_{30}$ 142.66: $\mathrm{C}_{23}, 144.39: \mathrm{C}_{1}, 144.87: \mathrm{C}_{36}, 146.07: \mathrm{C}_{16}, 149.21: \mathrm{C}_{9}$, 15.92: $\mathrm{C}_{13}, 159.20: \mathrm{C}_{5},{ }^{13} \mathrm{C}-\mathrm{DEPT} ;-43.56: \mathrm{CH}_{2} \mathrm{C}_{8},-64.56: \mathrm{CH} \quad \mathrm{C}_{7},-69.16: \mathrm{O}-\mathrm{CH}_{2} \mathrm{C}_{22}$, 71.69: $\mathrm{O}-\mathrm{CH}_{2} \quad \mathrm{C}_{29}, 112.11: \mathrm{C}_{4}, 113.31: \mathrm{C}_{37,41}, 114.05: \mathrm{C}_{6}, 114.94: \mathrm{C}_{12}, 118.09: \mathrm{C}_{2}$, 118.67: $\mathrm{C}_{17}, 119.13: \mathrm{C}_{15}$, 127.12: $\mathrm{C}_{18}$, 127.34: $\mathrm{C}_{24,28}, 128: 01 \mathrm{C}_{26}, 128.63: \mathrm{C}_{25,27}$, 128.70: $\mathrm{C}_{31,35}, 128.89: \mathrm{C}_{32,34}, 130.93: \mathrm{C}_{20}, 130.15: \mathrm{C}_{3}, 130.63: \mathrm{C}_{19}, 131.58: \mathrm{C}_{11}$. 


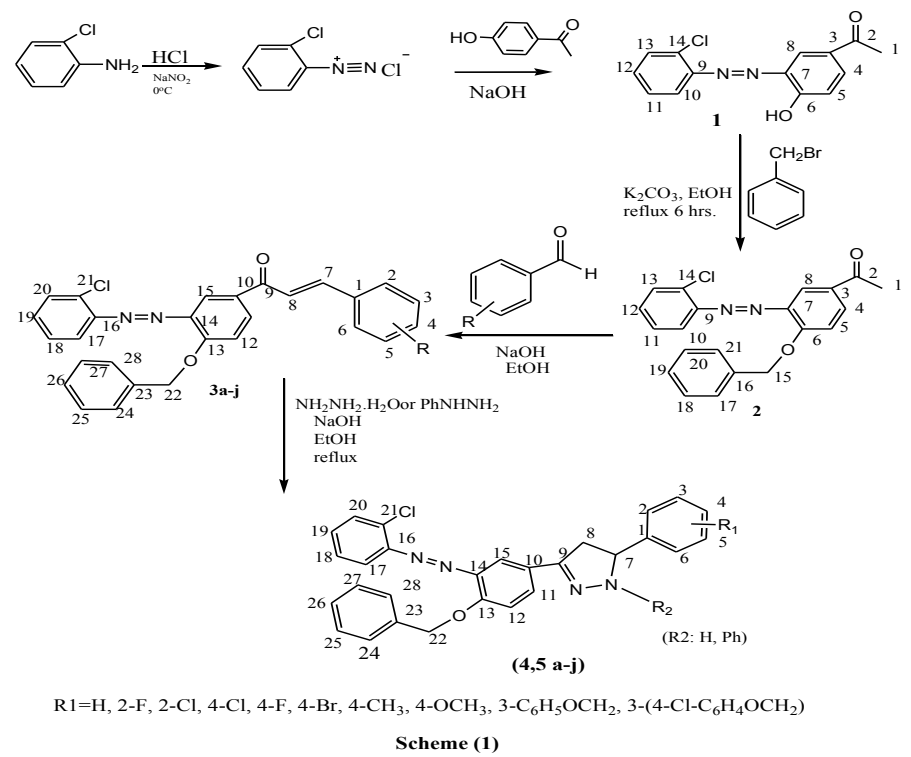

\section{Results and Discussion}

The synthesis of two new series of the target molecules 2-pyrazoline containing benzyloxy and azo-linkage side-chain is outlines in scheme (1). The skeleton of the synthesized compounds were confirmed by physical properties and spectroscopic methods like FT-IR, ${ }^{1} \mathrm{H}-\mathrm{NMR},{ }^{13} \mathrm{C}-\mathrm{NMR}$ and ${ }^{13} \mathrm{C}$-DEPT-135. The IR spectrum of compound (1) showed a broad band at $(3431) \mathrm{cm}^{-1}$ attributed to $(\mathrm{OH}) \operatorname{group}^{[30]}$, a characteristic $\mathrm{N}=\mathrm{N}$ band was assigned at $(1557) \mathrm{cm}^{-1}{ }^{[31]}$,two strong bands at $1679 \mathrm{~cm}^{-1}$ and $1607 \mathrm{~cm}^{-1}$ referring to carbonyl and carbon-carbon double bonds. The ${ }^{1} \mathrm{H}-\mathrm{NMR}$ spectrum of compound (1) shows a singlet at (2.68) ppm for three protons of $\mathrm{CH}_{3}$ attached to the carbonyl group, multiplet at (7.12- 8.06) and a singlet at (8.64) ppm for seven protons of the two phenyl rings and a distinct singlet signal at (12.25) ppm assigned to the hydroxyl group. ${ }^{13} \mathrm{C}$-NMR shows twelve singlet signals corresponding to twelve types of carbon in different chemical shifts. The IR spectrum of compound (2) shows the disappearance of a broad band at (3431) $\mathrm{cm}^{-1}$ for hydroxyl group of 4-hydroxyacetophenone ${ }^{[25]}$, and shifting the absorption band of carbonyl group from $1679 \mathrm{~cm}^{-1}$ to $1673 \mathrm{~cm}^{-1}$, is considered as a good evidence to benzyloxation processes, two strong bands at $\left(2927\right.$ and 2873) $\mathrm{cm}^{-1}$ equivalent to the $\left(-\mathrm{CH}_{2}-\right)$ group of 4-benzyloxy substrate. The ${ }^{1} \mathrm{H}-\mathrm{NMR}$ spectrum of compound (2) shows two singlet signals at (2.61 and 5.39) ppm belongs to the three protons of $\left(-\mathrm{CH}_{3}\right)$ attached to the carbonyl group and two protons of $\left(-\mathrm{O}-\mathrm{CH}_{2}-\right)$ respectively, a multiplet signals at (7.16-8.04) ppm, and a singlet signal at (8.45) ppm attributed to the twelve protons of aromatic rings. The ${ }^{13} \mathrm{C}-\mathrm{NMR}$ spectrum showed three singlets at $(26.38,71.28$ and 196.70$)$ ppm belongs to the carbon atom of $\left(-\mathrm{CH}_{3},-\mathrm{O}-\mathrm{CH}_{2}-\right.$, and carbonyl) with fourteen signals for fourteen carbons in aromatic regions. The ${ }^{13} \mathrm{C}$ DEPT-135 of compound (2) showed upward signal at $\delta(26.34)$ for tri-protonated carbon atom of $\left(-\mathrm{CH}_{3}\right)$ group and a downward signal at (71.34) corresponding to the diprotonated carbon atom $\left(-\mathrm{O}-\mathrm{CH}_{2}-\right)$ group with disappearance of non protonated carbon atoms . The FT-IR spectra of all chalcones (3a-j) showed the characteristic peaks of particular carbonyl functional groups ${ }^{[26]}$ in the region of $(1661-1653) \mathrm{cm}^{-1}$, the lowering 
of normal $(\mathrm{C}=\mathrm{O})$ frequency from $(1673) \mathrm{cm}^{-1}$ indicates the presence of $(\mathrm{C}=\mathrm{C})$ conjugated to carbonyl group (conjugated enones) ${ }^{[27]}$, table-4.

Table-4: Assignment of characteristic frequencies $\left(\mathrm{cm}^{-1}\right)$ of IR spectra for the prepared chalcones (3a-j) and pyrazolines (4 a-j), (5a-j) .

\begin{tabular}{|c|c|c|c|c|c|c|}
\hline \multirow{2}{*}{ Prod. } & \multirow{2}{*}{$\mathbf{R}$} & \multicolumn{2}{|c|}{ Chalcones (3 a-j) } & \multicolumn{2}{|c|}{ Pyrazolines (4 a-j) } & \multirow{2}{*}{\begin{tabular}{|c}
$\begin{array}{c}\text { Pyrazolines } \\
(5 \text { a-j })\end{array}$ \\
$\mathbf{C}=\mathbf{N}$
\end{tabular}} \\
\hline & & $\mathrm{C}=\mathrm{O}$ & $\mathrm{C}=\mathrm{C}$ & $\mathrm{C}=\mathrm{N}$ & N-H & \\
\hline $\mathrm{A}$ & $\mathrm{H}$ & 1659 & 1605 & 1605 & 3296 & 1597 \\
\hline B & $2-\mathrm{F}$ & 1602 & 1605 & 1603 & 3342 & 1598 \\
\hline $\mathrm{C}$ & $2-\mathrm{Cl}$ & 1659 & 1604 & 1606 & 3346 & 1597 \\
\hline $\mathrm{D}$ & $4-\mathrm{Cl}$ & 1661 & 1604 & 1606 & 3309 & 1598 \\
\hline $\mathrm{E}$ & $4-\mathrm{F}$ & 1661 & 1595 & 1604 & 3326 & 1597 \\
\hline $\mathrm{F}$ & $4-\mathrm{Br}$ & 1659 & 1604 & 1594 & 3335 & 1595 \\
\hline G & $4-\mathrm{CH}_{3}$ & 1661 & 1602 & 1603 & 3334 & 1597 \\
\hline $\mathrm{H}$ & $4-\mathrm{OCH}_{3}$ & 1653 & 1594 & 1606 & 3302 & 1597 \\
\hline I & $3-\mathrm{OCH}_{2} \mathrm{C}_{6} \mathrm{H}_{5}$ & 1660 & 1600 & 1602 & 3326 & 1598 \\
\hline $\mathrm{J}$ & $\begin{array}{c}3-\left(4-\mathrm{Cl}-\mathrm{C}_{6} \mathrm{H}_{4-}\right. \\
\left.\mathrm{CH}_{2} \mathrm{O}\right)\end{array}$ & 1660 & 1595 & 1600 & 3312 & 1597 \\
\hline
\end{tabular}

Table-5: Anti-bacterial activity of some prepared chalcones and pyrazolines with inhibition zone diameters in (mm) scale against $S$-aureus and $E$-coli bio-organisms.

\begin{tabular}{|c|c|c|}
\hline Product no. & E.coli $\mathbf{G}(-\mathbf{v e})$ & S-aureus \\
\hline 2 & 6 & 25 \\
\hline $3 \mathrm{a}$ & 8 & 25 \\
\hline $4 \mathrm{a}$ & 11 & 25 \\
\hline $5 \mathrm{a}$ & 25 & zero \\
\hline $3 \mathrm{e}$ & 9 & 25 \\
\hline $4 \mathrm{e}$ & 12 & 25 \\
\hline $5 \mathrm{e}$ & 18 & zero \\
\hline $3 \mathrm{f}$ & 8 & 25 \\
\hline $4 \mathrm{f}$ & 12 & 25 \\
\hline $5 \mathrm{f}$ & 18 & zero \\
\hline $3 \mathrm{j}$ & 10 & 25 \\
\hline $4 \mathrm{j}$ & 14 & 25 \\
\hline $5 \mathrm{j}$ & 16 & zero \\
\hline
\end{tabular}

The ${ }^{1} \mathrm{H}$-NMR spectra of chalcones, show characteristic doublet signals for $\alpha, \beta$ - protons at (8.18) $\mathrm{ppm}^{[28]}$, this deshielding refers to the effect of resonance of the phenyl rings that bonded to $\beta$ - carbon atom, but the $\left(\mathrm{CH}_{\alpha}\right)$ completely emerged with aromatic protons, it is 
hard to distinguish it at a certain number. The ${ }^{13} \mathrm{C}-\mathrm{NMR}$ spectra assignment of carbon atoms presented in chalcones moiety, show the characteristic peak is that related to the $\beta-\mathrm{C}$ atom nearly around (143 - 145) ppm which is more deshielded than that of $\alpha-\mathrm{C}$ atom approximately at (118) ppm by the mesomeric action of carbonyl group ${ }^{[29]}$. The ${ }^{13} \mathrm{C}$-DEPT135 appeared a characteristic downward signal approximately at (71) ppm corresponding to the di-protonated carbon $\left(\mathrm{C}_{22}\right)$ atom of the $\left(-\mathrm{O}-\mathrm{CH}_{2}-\right)$ group, and two characteristic monoprotonated carbon, at (118 and143-145) ppm belongs to $\left(\mathrm{C}_{\alpha}\right.$ and $\left.\mathrm{C}_{\beta}\right)$ atoms respectively, and the other peaks return to Ar-C atoms. In the IR spectra of pyrazolines (4 a-j) table-4, fig.(1) exhibited a characteristic band at $(3296-3346) \mathrm{cm}^{-1}$ due to $\mathrm{N}-\mathrm{H}$ stretching ${ }^{[30]}$, also pyrazolines (5a-j) appeared strong band at (1594-1606) $\mathrm{cm}^{-1}$ for $\mathrm{C}=\mathrm{N}$ stretching vibration, beside the appearance of above bands, the most important evidence for the formation of 2pyrazoline is the disappearance of carbonyl group band at $(1653-1661) \mathrm{cm}^{-1}$ special for the chalcone moiety. The ${ }^{1} \mathrm{H}-\mathrm{NMR}$ spectra of pyrazolines showed characteristic signals corresponding to proton of $\mathrm{C}_{8}$ and $\mathrm{C}_{7}$ of 2-pyrazoline ring; they form a typical $\mathrm{ABX}$ system confirming the nonequivalence of protons at $\mathrm{C}_{8}{ }^{[31]}$. It causes to appearance of three doublet to doublet (dd) signals approximately at (3, 3.5 and 4.7) for each compound, fig. (2) .

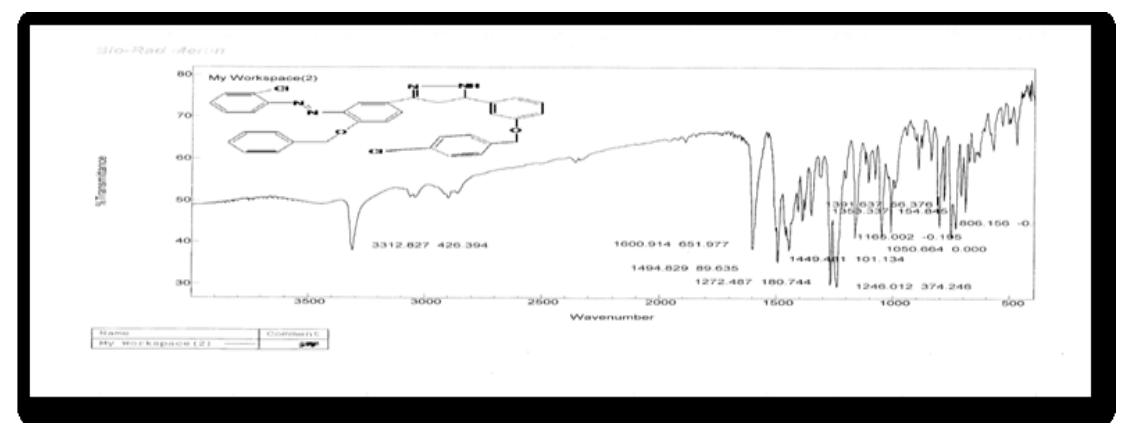

Figure 1: IR spectrum of compound (4j).

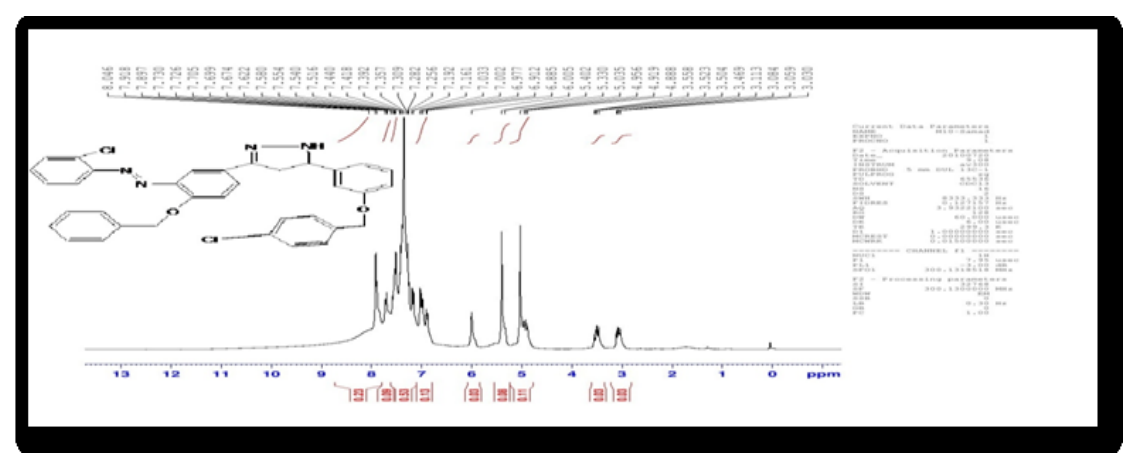

Figure 2: ${ }^{1} \mathrm{H}-\mathrm{NMR}$ spectrum of compound $(4 \mathrm{j})$.

The ${ }^{13} \mathrm{C}$-NMR spectra of pyrazolines, showed three signals approximately at, (41.5, 63.79 and 71.66) belongs to $\left(\mathrm{C}_{8}, \mathrm{C}_{7}\right.$ and $\left.\mathrm{C}_{22}\right)$ for pyrazoline carbon and $\mathrm{O}-\mathrm{CH}_{2}$ groups respectively, and the other peaks between (112 and 164) ppm approximately attributed to 
aromatic carbon atoms fig(3). The ${ }^{13} \mathrm{C}$-DEPT-135 of pyrazolines exhibited tree downward signals at $(40,64$ and 71$) \mathrm{ppm}$ approximately indicated to the di-protonated carbon atoms of pyrazoline ring and two $-\mathrm{OCH}_{2}$ - group respectively, also the appearance of other upward signals corresponding to mono-protonated carbon atoms, Fig.(4)

\section{Antibacterial Activity:}

The synthesized compounds were screened for antimicrobial activity against two types of bacteria Escherichia coli (gram negative ) and Staphylococcus aureus (gram positive ) by using the cup-plate agar diffusion method with $\mathrm{KBr}$ disc of compounds .The prepared discs were placed on the surface of the cultured media with each of the two bacteria's and incubated for 24 hours at $37^{\circ} \mathrm{C}$ and the results were monitored by measuring the zones of inhibition in $\mathrm{mm}$. The screening results are listed in table (5) and they show that most of the prepared compounds are sensitive against both types of test organisms in different activities.

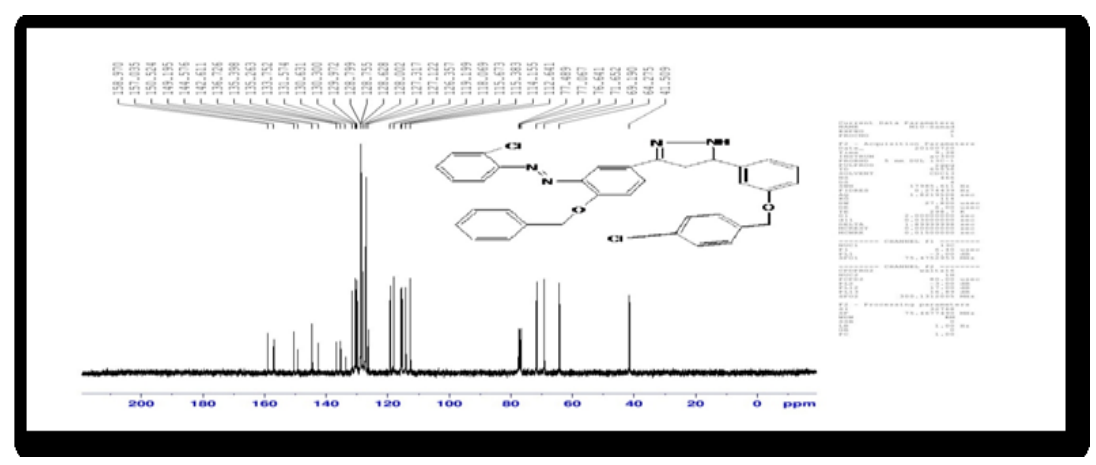

Figure 3: ${ }^{13} \mathrm{C}-\mathrm{NMR}$ spectrum of compound $(4 \mathrm{j})$.

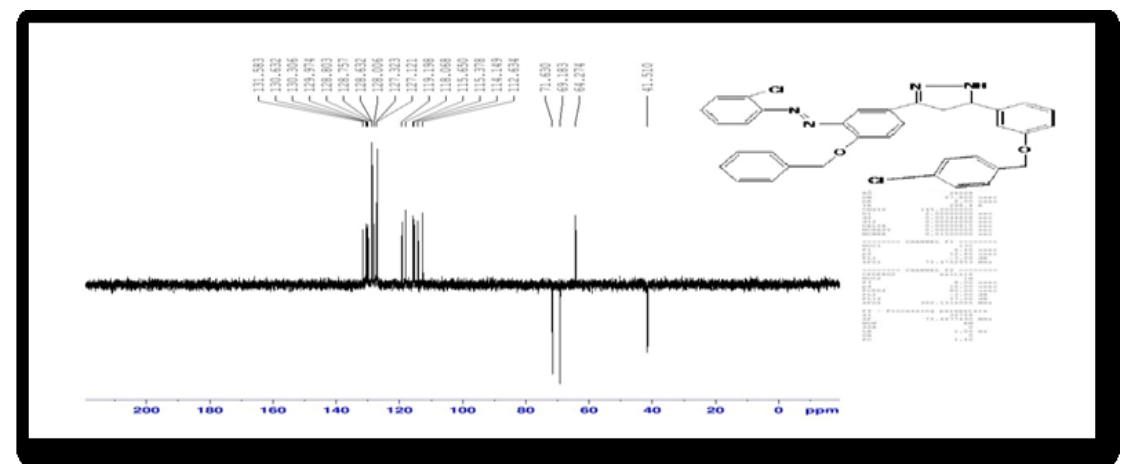

Figure 4: ${ }^{13} \mathrm{C}$-Dept spectrum of compound $(4 \mathrm{j})$.

\section{References}

1. Farouq E.H, Essa I. A and Mohanad H.M. M, Zanco, 2010,22,6, 78-87

2. LI J. P , LIU P and Wang. Y. L. Chinese Chemical Letters , 200314, 7, $677-680$

3. Jarrahpour A. A, Motamedifar M , Pakshir K, Hadi N. and Zarei M, Molecules, (2004). 9, 815-824

4. Abdullah, M. A, JKAU Sci, 2000, 12, 69-76 
5. Karukstis K. K. , Perelman L. A. and Wong W. K. Langmuir 2002, 18, 10363-10371

6. Murphy A. R, John P. St, and Kaplan D.L. Biomaterials 2008, 29 , 2829-2838

7. Zhou M, and He J, Electrochimica Acta, 2007, 53, 1902.

8. Prasad Y. R, Kumar P. P., and Rao A. S., E-Journal of Chemistry, 2008, 5, 1, 144

9. Cherkupally S. R, Gurrala P. R., Adki N. and Avula S, Org. Commun., 2008, 1, 4, 84

10. Guo C, X., Du H, McKerrow J. H. and Cohen F. E , cruzi, Chinese Chemical Letters, 2002, 13, 11, 1043,

11. Azarifar D. and Shaebanzadeh M, molecules, 2002, 7, 885

12. Goda F. E, Maorouf A. R. and EL-Bendary E. R . Saudi Pharmaceutical Journal, 2003,11, 3, 111

13. Li R, Kenyon G. L. and Cohen F. E, J. Med. Chem., 1995,38, 5031

14. Asiri A. M and Khan S. A, molbank, 2009, 636, 1

15. Arty I. S, Timmerman H, Samhoedi M, Sugiyanto S. and Goot V. D , Europ. J. Med. Chem., 2000, 35,449

16. Prasad Y. R, Kumar P. P, Kumar and P. R and Rao A. S, E-Journal of Chemistry, 2008, 5, 1, 144

17. Ceylan M. and Gezegen H, Turk J Chem. 2008, 32, 55.

18. Nagaraj A. and Reddy C. S, J. Iran. Chem. Soc, 2008, 5, 2, 262.

19. Levai A. and Jeko J, ARKIVOC, 2005 (x), 199

20. Rahaman S.A, Prasad Y.R, Bhuvaneswari K. and Kumar P, Int.J. Chem Tech. Res., 2010, 2, 1, 16

21. Hamzaçebi M. C, Rollas S., Kucukguzel S. G., and Kaymakcioglu B. K., ARKIVOC, 2008 (xii), 188.

22. Ngaini Z., Haris-Fadzillah S. M., Hussain H. and Kamaruddin,K., World J. Chemistry, $2009,4,1,9$.

23. Kalirajan R, Sivakumar S.U., Jubie S., Gowramma B. and Suresh B, International Journal of ChemTech Research, 2009,1, 1, 27

24. Guo H. M, Wang L.T, Zhang J. Zhao P.S and Jian F. F, , Molecules, 2008, 13, 2039.

25. Field L. D, Stern hell S. and Kalman J. R.. Organic Structures from Spectra, $3^{\text {rd }}$ eddition, John Wiley and Sons, 2005, 64-68.

26. Chaczatrian K., Chaczatrian G, Danel A, and Tomasik P, , ARKIVOC, 2001 (vi), 67.

27. Kumar J. A. and Sondu, S., Indian journal of Chemistry, $2007,46 \mathrm{~A}, 1792$.

28. Curey F., Organic Chemistry, $7^{\text {th }}$ edition, 2009, 753-759.

29. Gurubasavaraja P.M. and Agasimundin Y.S., Acta Pharmaceutica Sciencia, 2008 , 50, 197,

30. Ali S M. and Yar M. S , Acta poloniae Pharmaceutica-Drug Research, 2007, 64, 2, 139-146.

31. Al-Issa S. A and Al-Andis N, , J. Saudi Chem. Soc, 2005, 9, 3, 689. 


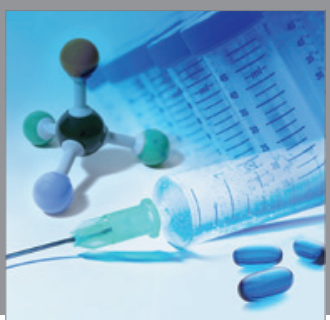

International Journal of

Medicinal Chemistry

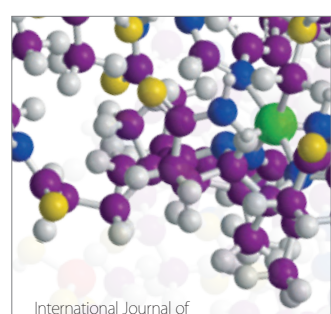

Carbohydrate Chemistry

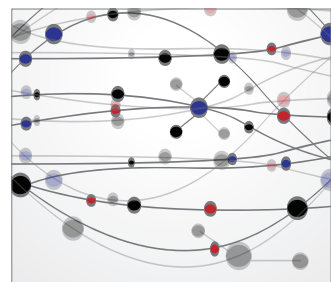

The Scientific World Journal
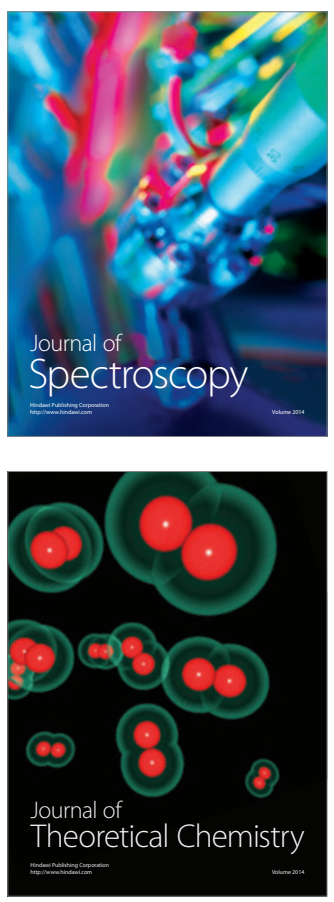
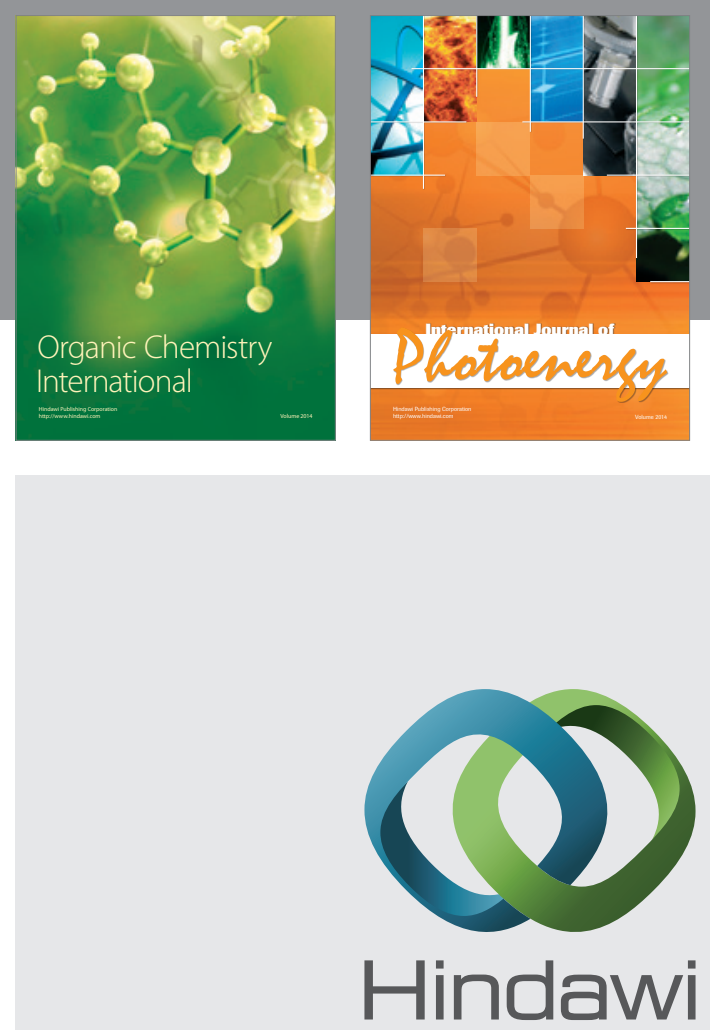

Submit your manuscripts at

http://www.hindawi.com
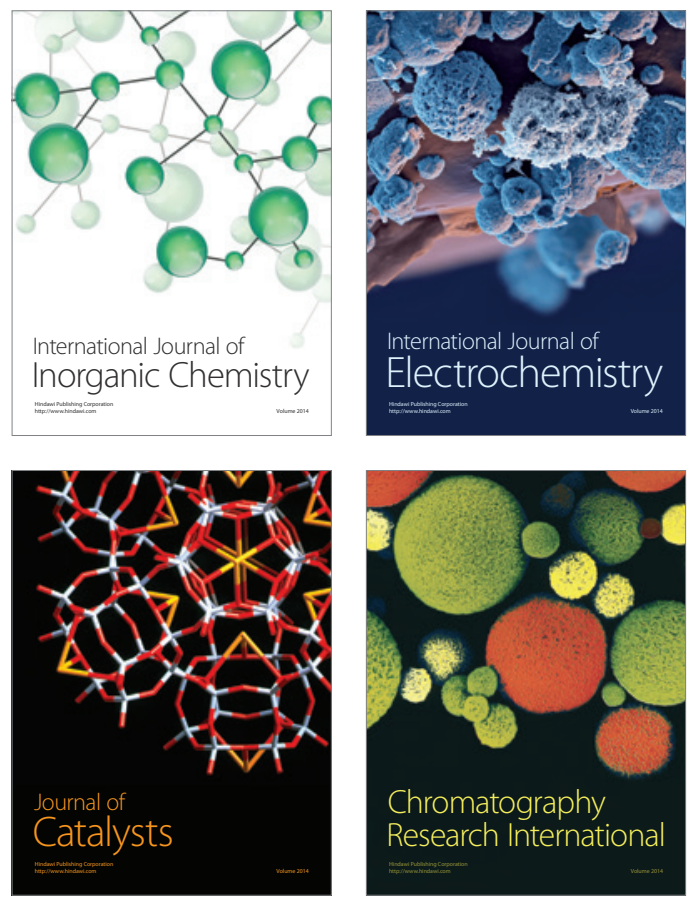
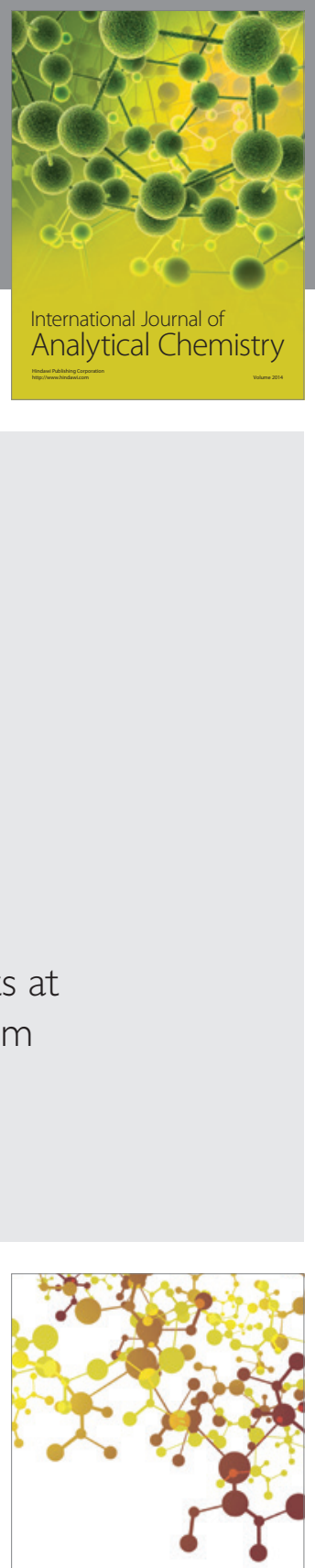

Journal of

Applied Chemistry
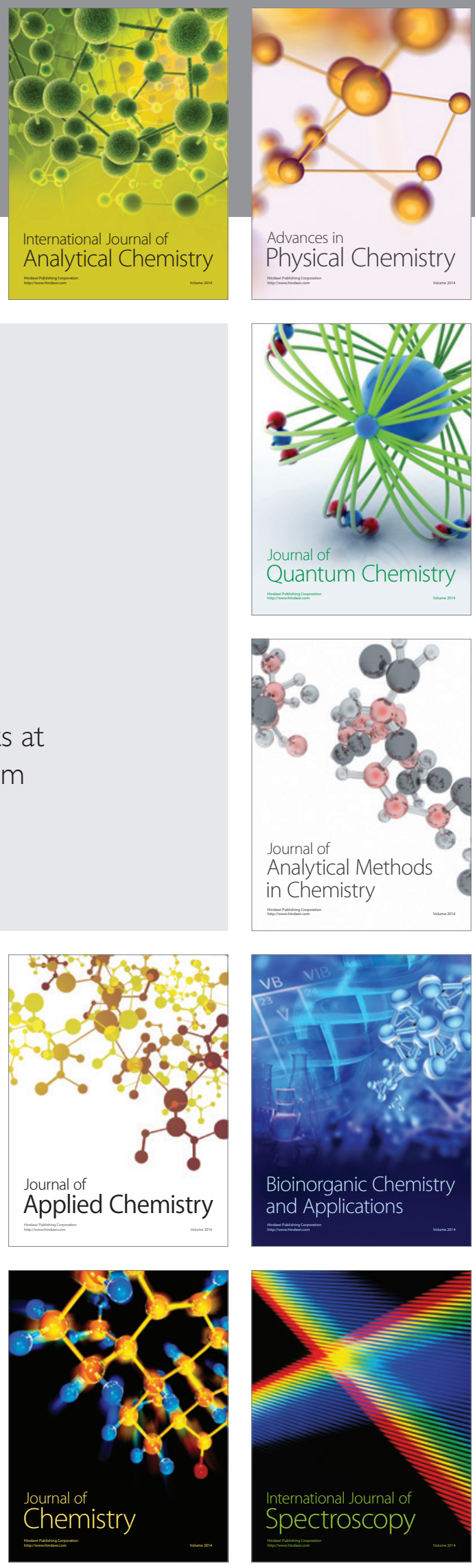